\title{
Vector analysis of ice-fabric data
}

\author{
M. G. Ferrick and K.J. Claffey \\ U.S. Army Cold Regions Research and Engineering Laboratory, Hanover, New Hampshire 03755-1290, U.S.A.
}

\begin{abstract}
The mechanical properties of ice are strongly affected by crystal texture and $c$-axis alignment. In this paper, we develop a general quantitative method for analysis of uniaxial crystal-orientation data. These data are represented as unit vectors from the origin with end points on the surface of a unit sphere. An orthogonal least-squares error measure is used to develop equations that define the closest plane and line through the data. The resulting eigenvalue problem is identical to that obtained by other investigators using different methods. However, here we identify an implicit assumption in the method, and observe that the error measure represents physical distance and quantifies the goodness of fit to the data of idealized structures. Also, a method is developed to transform the data and the results for viewing on Schmidt nets drawn in the best plane and the predominant basal plane of a sample, in addition to the standard $x y$-plane. Applications of the analysis to sea-ice samples include both numerical and Schmidt-net presentations of results.
\end{abstract}

\section{INTRODUCTION}

Environmental conditions at the time of ice formation largely determine its structure. Ice crystals are uniaxial and the optic axis corresponds to the $c$-axis. Random $c$ axis orientation is commonly observed near the top surface of newly formed sea ice in the Arctic. Once a cover has formed, the ice structure is characterized by long vertical columns that extend downward in the growth direction of the ice sheet, a result of quiescent, unidirectional growth. Under these conditions, a selective growth process occurs and the $c$-axes of the crystals become primarily oriented in the horizontal plane of the ice sheet (Weeks and Ackley, 1982). In the presence of a predominant current direction, strong $c$-axis alignment develops in the direction of the current, with generally decreasing scatter as ice thickness increases (Weeks and Gow, 1978), and this ice structure causes anisotropic material behavior in all directions. Uniaxial compression data on first-year sea ice (Wang, 1979; Richter-Menge and others, 1987) have indicated a strong dependence of peak compressive strength on $c$-axis alignment and on the angle between an applied load and the dominant $c$-axis direction. Therefore, to interpret data from mechanical property tests, we must define the relative orientation and alignment of the ice fabric.

The techniques used in the analysis of ice fabrics were originally developed in structural petrology (see, for example, Knopf and Ingerson, 1938; Fairbairn, 1949; Turner and Weiss, 1963). Crystal-orientation measurements usually involve optical measurements of the $c$-axis orientations. Langway (1958) described techniques for obtaining ice-crystal $c$-axis orientation data using a Rigsby universal stage. One orientation measurement is made for each ice crystal in a sample, and these data are plotted on a Schmidt equal-area net that represents the surface of a hemisphere of unit radius. The points on the net comprise an orientation diagram that depicts the relative spatial concentration of the data. An orientation diagram may indicate a random or a patterned structure, depending on the dominant features of the diagram. A random fabric refers to a homogeneous distribution of the data and represents an isotropic material. In this configuration, there is an equal probability of finding points in equal-area elements anywhere on the net. In contrast, the most significant feature of an anisotropic material is the preferred orientation indicated by the grouping of points on the net. A "girdle" corresponds to data that are distributed along a great circle of the net, indicating a preferred planar orientation of the $c$-axes. The pole of this great circle is termed the girdle axis. An area of highly concentrated data points (point maximum) indicates a linear-preferred orientation of the crystals in the fabric. The statistical significance of the orientation diagram increases if the main features are reproducible in different comparable samples from the same homogeneous body.

Pearson (1901) used statistical arguments to develop the equation of a line or plane that provided the closest fit to points in space when all variables contain error. The solution depends on knowledge of the means, standard deviation and correlations of the variables. A significant result was that the plane of best fit contains the line of best fit. Watson (1966) presented a matrix of sums of direction cosines of vectors representing crystal orientation in a Cartesian coordinate system. The reasoning presented was that the greatest moment of inertia of the points would be about the eigenvector corresponding to the minimum eigenvalue of the matrix. Mardia (1972) used the same reasoning to obtain this matrix and interpret the results. Both Watson and Mardia proposed distributions for the data on the sphere, and developed statistical 
analyses based on these assumptions. Diggle and Fisher (1985) described a program that computed these eigenvalues and eigenvectors and quantitatively contoured spherical data. The analysis of ice-fabric diagrams has been largely visual, frequently based on approximate data-concentration contours drawn on the net. However, Herron and Langway (1982) applied the eigenvalue/ eigenvector method of Mardia (1972) to study various fabric types, including small-circle girdles and multimaxima patterns. The results were interpreted qualitatively and it was not clear whether any fabrics could be quantitatively assessed by this method.

In this paper, we will seek a plane passing through the origin that minimizes the sum of the squared normal distances from the data, and obtain the dominant $c$-axis orientation in this plane. A detailed derivation from simple geometric arguments is developed, yielding leastsquares equations that minimize the orthogonal distance between the data and the best line and plane. The eigenvalue problem that results is the same as that obtained by Pearson, Watson and Mardia. We identify an implicit assumption in this method of equal-measurement uncertainty in each coordinate at all points on the sphere. Normalized eigenvalues provide quantitative measures of physical distance of the data from the plane and line, specifying the directional characteristics of the $c$ axes of crystals in a sample. Mean angular measures of variability are also developed. This analysis clearly indicates the fabrics that are well-described by the eigenvalue/eigenvector method, and provides a framework for developing related quantitative methods for other fabric types. We demonstrate the capabilities of the analysis on data sets representing samples of first-year sea ice. The results are viewed on special Schmidt nets that represent data hemispheres defined by the best plane and the predominant basal plane, in addition to the $x y$-plane.

\section{MEASUREMENT ERRORS AND DATA FITTING}

Measurement of the $c$-axis orientation of an ice crystal with a Rigsby universal stage provides an azimuth angle, the direction of inclination as right or left and the type of measurement as polar or equatorial. From these data, we obtain a pair of angles $(\theta, \phi)$ that define the orientation (Ferrick and Claffey, 1992). The line representing each crystal in the sample is plotted through the origin of a unit sphere. Each of these lines intersects the surface of the sphere at one point in each hemisphere and the lower hemisphere is traditionally studied. Our analysis represents the $c$-axis of each ice crystal as a three-dimensional unit vector from the origin in the half-space below the $x y$ plane, yielding an array of points on the surface of a hemisphere of unit radius $\rho$. The three-dimensional Cartesian coordinates of each point on this hemisphere (Fig. 1) are obtained directly from the spherical cordinates $(\rho, \theta, \phi)$ as

$$
\begin{aligned}
& x=\rho \sin \phi \cos \theta \\
& y=\rho \sin \phi \sin \theta \\
& z=\rho \cos \phi .
\end{aligned}
$$

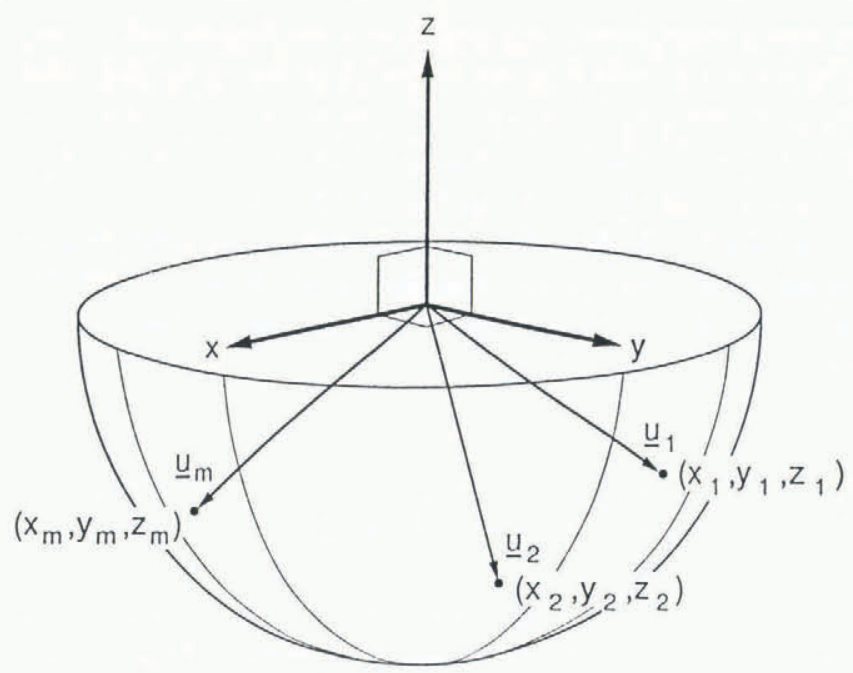

Fig. 1. Sketch of unit vectors $1,2 \ldots, m, \ldots \mathcal{N}$ representing the $c$-axis orientations of ice crystals in a sample. The hemisphere, $z \leq 0$, is shown by convention.

The relative uncertainties in $x, y$ and $z$ caused by measurement error must be understood to identify an appropriate method for fitting the ice-fabric data. Langway (1958) listed several sources of error in the measurement of $c$-axis orientation, and indicated that the errors in azimuth and inclination should each be less than $5^{\circ}$. We observe that the same statement holds for errors in $\theta$ and $\phi$. Taking these errors as random and each of magnitude $\beta$, we have from Equations (1) that

$$
\begin{aligned}
& x=\sin (\phi \pm \beta) \cos (\theta \pm \beta) \\
& y=\sin (\phi \pm \beta) \sin (\theta \pm \beta) \\
& z=\cos (\phi \pm \beta) .
\end{aligned}
$$

Expanding Equations (2) and grouping terms according to the error, we obtain

$$
\begin{aligned}
& x=\sin \phi \cos \theta \cos ^{2} \beta+\sin \beta(A \cos \beta+B \sin \beta) \\
& y=\sin \phi \sin \theta \cos ^{2} \beta+\sin \beta(C \cos \beta+D \sin \beta) \\
& z=\cos \phi \cos \beta+\sin \beta(E),
\end{aligned}
$$

where $A, B, C, D$ and $E$ are composed of sines and cosines of $\phi$ and $\theta$. The relationships given in Equations (1) are contained in Equations (3), but modified by errors of $\beta$. An error in $\theta$ only affects $x$ and $y$. The resulting uncertainty in each coordinate is periodic, depending on $\theta$, and out of phase with the other. The amplitude of these errors approaches zero near the pole and a maximum at the equator. Errors in $\phi$ affect all three Cartesian coordinates. The uncertainty in $z$ is larger near the equator and smaller near the pole than the larger of $x$ and $y$. Uncertainties in $x$ and $y$ resulting from errors in $\phi$ again vary individually with $\theta$, displaying maximum amplitudes near the pole and approaching zero near the equator.

Classical least-squares methods require a dependent variable but the coordinates of each $c$-axis are all independent. Reed (1989) presented a method for fitting a line to points in the plane when both coordinates of these points are independent and uncertain due to measurement error. The method allows the errors in the fit to be weighted according to the relative uncertainties 
in the measurement of $x$ and $y$. The measurement errors discussed above could be considered by extending this method to three dimensions. However, we note for small $\beta$ that $\cos \beta \approx \cos ^{2} \beta \approx 1$ and $\sin \beta \approx 0$, indicating that errors in $x, y$ and $z$ resulting from measurement errors are relatively small. As a first approximation, we will choose equal weighting in each direction at every point and the best-fit line and plane will minimize the perpendicular distances from the data.

\section{DETERMINATION OF THE BEST PLANE BY ORTHOGONAL LEAST SQUARES}

The unit vectors representing the $c$-axis orientation of each crystal in a sample have a common point at the origin of the unit sphere. The problem we consider here is to find the plane of best fit to fabric data that contains the origin, and to provide quantitative measures of the quality of the fit. The form of the equation of a plane through the origin is

$$
f(x, y, z)=A x+B y+C z=0 .
$$

We choose the function $F\left(x, y, z ; c_{1}, c_{2}, c_{3}\right)$ with this same form and depending linearly on parameters $c_{1}, c_{2}, c_{3}$ as

$$
F\left(x, y, z ; c_{1}, c_{2}, c_{3}\right)=c_{1} \phi_{1}+c_{2} \phi_{2}+c_{3} \phi_{3}=\sum_{i=1}^{3} c_{i} \phi_{i}=0
$$

where $\phi_{1}=x, \phi_{2}=y, \phi_{3}=z$ are a specified set of mutually orthogonal functions and the $c_{i}$ are unknowns to be determined. The pole of a plane is the point of intersection $\mathrm{P}$ with the hemisphere of a line through the origin normal to the plane. The unit normal $\mathbf{n}$ to the plane of best fit has the form

$$
\mathbf{n}=c_{1} \mathbf{i}_{1}+c_{2} \mathbf{i}_{2}+c_{3} \mathbf{i}_{3}=c_{j} \mathbf{i}_{j}
$$

where $\mathbf{i}_{1}, \mathbf{i}_{2}$ and $\mathbf{i}_{3}$ are unit vectors in the $x, y$ and $z$ directions, respectively, and repeated indices indicate summation.

A unit vector in three-dimensional space represents each crystal, and the total number of crystals in a sample $\mathcal{N}$ used to fit the plane should be much larger than 3 . The unit vector representing the $m$ th crystal (Fig. 2) is

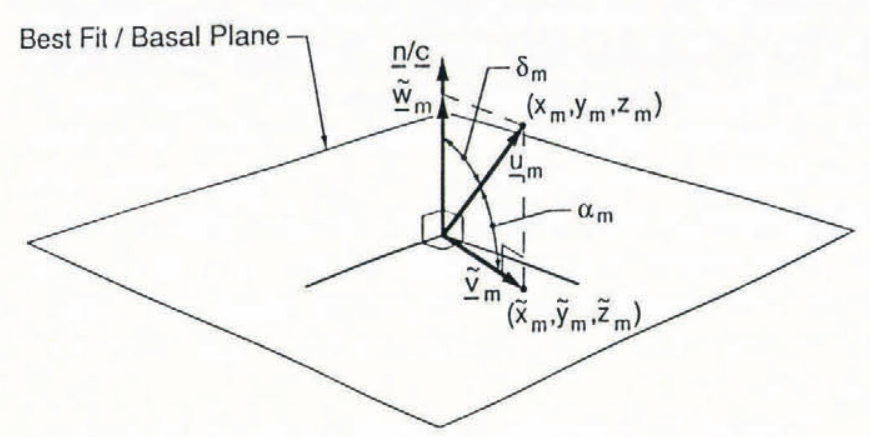

Fig. 2. Sketch of the unit vector $\mathbf{u}_{m}$ representing the mth crystal in an ice sample and its projections $\tilde{\mathbf{v}}_{m}$ on to the best-fit plane or the basal plane, and $\tilde{\mathbf{w}}_{m}$ on to $\mathbf{n}$ or $\mathbf{c}$ the unit normal vector to the corresponding plane. The angles between $\mathbf{u}_{m}$ and the best (plane, line) are $\left(\alpha_{m}, \delta_{m}\right)$, respectively.

$$
\begin{aligned}
\mathbf{u}_{m} & =x_{m} \mathbf{i}_{1}+y_{m} \mathbf{i}_{2}+z_{m} \mathbf{i}_{3} \\
& =\phi_{1_{m}} \mathbf{i}_{1}+\phi_{2_{m}} \mathbf{i}_{2}+\phi_{3_{m}} \mathbf{i}_{3}=\phi_{j m} \mathbf{i}_{j} .
\end{aligned}
$$

This vector intersects the unit sphere at $\left(x_{m}, y_{m}, z_{m}\right)$ and we can evaluate $F\left(x_{m}, y_{m}, z_{m} ; c_{1}, c_{2}, c_{3}\right)=F_{m}$ with Equation (5) as

$$
F_{m}=c_{1} x_{m}+c_{2} y_{m}+c_{3} z_{m} .
$$

The vector $\tilde{\mathbf{w}}_{m}$ is the projection of $\mathbf{u}_{m}$ on to $\mathbf{n}$, representing the normal vector from the plane to the point $\left(x_{m}, y_{m}, z_{m}\right)$

$$
\tilde{\mathbf{w}}_{m}=\left(u_{m} \cdot n\right) \mathbf{n}
$$

where $\left(u_{m} \cdot n\right)$ is a scalar product between unit vectors. Throughout this development, the notation $(\sim)$ over a vector indicates that it does not have unit length. The normal distance $d_{m}$ from point $m$ to the plane is

$$
d_{m}=\left(u_{m} \cdot n\right)=c_{1} x_{m}+c_{2} y_{m}+c_{3} z_{m}
$$

and we observe that

$$
\begin{aligned}
& \left|d_{m}\right|=\left|\tilde{\mathbf{w}}_{m}\right| \leq 1, \\
& \tilde{\mathbf{w}}_{m}=d_{m} \mathbf{n} .
\end{aligned}
$$

The sign of $d_{m}$ distinguishes distances on opposite sides of the plane.

The orthogonal projection of $\mathbf{u}_{m}$ in the best-fit plane is represented by the vector $\tilde{\mathbf{v}}_{m}$, and

$$
\tilde{\mathbf{v}}_{m}=\mathbf{u}_{m}-\tilde{\mathbf{w}}_{m}=\tilde{\mathbf{x}}_{m} \mathbf{i}_{1}+\tilde{\mathbf{y}}_{m} \mathbf{i}_{2}+\tilde{\mathbf{z}}_{m} \mathbf{i}_{3}=\tilde{\phi}_{j m} \mathbf{i}_{j}
$$

This vector joins the origin and the point $\tilde{x}_{m}, \tilde{y}_{m}, \tilde{z}_{m}$, the orthogonal projection of $\left(x_{m}, y_{m}, z_{m}\right)$ in the plane. The vector $\tilde{\mathbf{v}}_{m}$ is unique and the best approximation of $\mathbf{u}_{m}$ in the plane. An equivalent statement is that the closest point in the plane to $\left(x_{m}, y_{m}, z_{m}\right)$ is $\left(\tilde{x}_{m}, \tilde{y}_{m}, \tilde{z}_{m}\right)$. From Equation (12), we can determine $\tilde{x}_{m}, \tilde{y}_{m}, \tilde{z}_{m}$ as

$$
\begin{aligned}
& \tilde{x}_{m}=x_{m}-d_{m} c_{1} \\
& \tilde{y}_{m}=y_{m}-d_{m} c_{2} \\
& \tilde{z}_{m}=z_{m}-d_{m} c_{3}
\end{aligned} \quad \text { for } m=1,2, \ldots, N
$$

or

$$
\tilde{\phi}_{i m}=\phi_{i m}-d_{m} c_{i} \quad \text { for } i=1,2,3
$$

where $\phi_{i m}$ and $\tilde{\phi}_{i m}$ represent elements of $\mathcal{N}$-dimensional vectors $\phi_{i}$ and $\tilde{\phi}_{i}$, respectively.

We want to choose the $c_{i}$ values that specify the plane through the origin with normal distances $\left|d_{m}\right|$ between the points representing the $\mathcal{N}$ crystals in a sample and the plane that are as small as possible. The $\mathcal{N}$-vector $\mathbf{d}=\left(d_{1}, d_{2}, \ldots, d_{N}\right)^{T}$ represents the individual normal distances from the data to the plane, where $T$ indicates the transpose. Minimum normal distance to the plane is equivalent to maximum length of $\tilde{\mathbf{v}}_{m}$, the projection of $\mathbf{u}_{m}$ on to the plane. We will seek a least-squares fit and define $E$ as a function of the unknown coefficients

$$
E\left(c_{1}, c_{2}, c_{3}\right)=\sum_{m=1}^{N}\left|\tilde{\mathbf{v}}_{m}\right|^{2}=\sum_{m=1}^{N} \sum_{i=1}^{3}\left(\tilde{\phi}_{i m}\right)^{2} .
$$

Because we consider data projected on to a plane, the 
hemisphere in which the data appears is arbitrary. The sum of the squares of the lengths of $\tilde{\mathbf{v}}_{m}$ will be maximized and the sum of the squared distances from the plane $d_{m}$ will be minimized where the gradient of $E$ vanishes.

$$
\nabla E\left(c_{1}^{*}, c_{2}^{*}, c_{3}^{*}\right)=0
$$

with $c_{i}^{*}$ indicating coefficients of the plane of best fit.

We now perform the differentiation indicated in Equation (15) with respect to $c_{j}$, where each choice of $j$ yields a scalar equation of the form

$$
\frac{\partial E}{\partial c_{j}}=2 \tilde{\phi}_{i m} \frac{\partial \tilde{\phi}_{i m}}{\partial c_{j}}=0 .
$$

From Equation (13) we obtain

$$
\frac{\partial \tilde{\phi}_{i m}}{\partial c_{j}}=-\left(d_{m} \delta_{i j}+c_{i} \frac{\partial d_{m}}{\partial c_{j}}\right)
$$

where $\delta_{i j}$ is the Kronecker delta. We observe from Equation (10) that $d_{m}=c_{k} \phi_{k m}$, and

$$
\frac{\partial d_{m}}{\partial c_{j}}=\phi_{k m} \delta_{j k}=\phi_{j m} \text {. }
$$

Inserting Equations (13), (17) and (18) into Equation (16), we obtain the normal equations as

$$
\left(\phi_{i m}-d_{m} c_{i}\right)\left(d_{m} \delta_{i j}+c_{i} \phi_{j m}\right)=0
$$

or expanding and re-arranging as

$$
\phi_{j m} d_{m}\left(1-c_{i}^{2}\right)+\phi_{i m} \phi_{j m} c_{i}=d_{m}^{2} c_{j} .
$$

Finally, because $\mathbf{n}$ is a unit vector and $i$ is a summation index, $\left(1-c_{i}^{2}\right)=0$ and Equation (19) becomes

$$
\phi_{i m} \phi_{j m} c_{i}=d_{m}^{2} c_{j} \text {. }
$$

In Equation (20) we observe that $d_{m}^{2}=\lambda$, a constant, yielding an eigenvalue problem,

$$
A c=\lambda c
$$

where each term $a_{i j}$ of the $3 \times 3$ symmetric matrix $A$ is

$$
a_{i j}=\phi_{i m} \phi_{j m}=\phi_{i}^{T} \cdot \phi_{j} \text {. }
$$

The eigenvalue $\lambda$ is the sum of the squared normal distances of the data from the corresponding plane. Equation (21) in homogeneous form indicates that nontrivial solutions exist if and only if

$$
\operatorname{det}(A-\lambda I)=0 \text {. }
$$

The determinant given in Equation (23) yields a cubic equation called the characteristic polynomial

$$
\lambda^{3}+p \lambda^{2}+q \lambda+r=0 \text {. }
$$

Following Beyer (1987), we obtain the solution as

$$
\lambda=\left\{\begin{array}{l}
m \cos \beta-p / 3 \\
m \cos (\beta-2 \pi / 3)-p / 3 \\
m \cos (\beta-4 \pi / 3)-p / 3
\end{array}\right.
$$

where

$$
\begin{aligned}
\beta & =\frac{1}{3} \cos ^{-1}(3 b / a m) \\
m & =2 \sqrt{-a / 3} \\
a & =\frac{1}{3}\left(3 q-p^{2}\right) \\
b & =\frac{1}{27}\left(2 p^{3}-9 p q+27 r\right) .
\end{aligned}
$$

As $p, q$ and $r$ are real, the eigenvalues will also be real. Because $A$ is a real symmetric matrix, it is similar to a diagonal matrix $B$ composed of the eigenvalues of $A$, and therefore the eigenvalues of $A$ are real. Similar matrices have the same trace and the same determinant. The trace of a matrix is the sum of the elements on the principal diagonal. The elements $a_{11}, a_{22}$ and $a_{33}$ of matrix $A$ represent the sum of the squares of the distances between the data and the three planes defined by the coordinate axes. As each crystal is represented by a unit vector,

$$
\operatorname{tr}(\mathrm{A})=\sum_{i=1}^{3} a_{i i}=N=\operatorname{tr}(\mathrm{B})=\sum_{i=1}^{3} b_{i i}=\sum_{i=1}^{3} \lambda_{i}
$$

indicating that the sum of the eigenvalues is $\mathcal{N}$, the total number of crystals in the sample. The determinants of $A$ and $B$ are the product of the eigenvalues. The eigenvalues of $A$ are non-negative if $A$ is positive semi-definite; that is, $x^{T} \mathrm{~A} x \geq 0$ for any $x$. Since the eigenvalues represent sums of squared distances, $\lambda_{i} \geq 0$ and matrix $A$ is positive semidefinite. We will designate the eigenvalues in increasing order according to magnitude as $\lambda_{1} \leq \lambda_{2} \leq \lambda_{3}$, and define normalized eigenvalues as

$$
\lambda_{i}^{\prime}=\frac{\lambda_{i}}{N}
$$

The normalized eigenvalues give the mean squared normal distance between the points on the unit sphere defined by the unit vectors and the plane normal to the corresponding eigenvector. These eigenvalues provide a measure of the fit that is equivalent to the variance in classical dependent variable least-squares methods.

Eigenvectors of a real symmetric matrix corresponding to different eigenvalues are orthogonal and, because the eigenvalues are real, the eigenvectors can be taken to be real. The vector $\mathbf{v}$ is an eigenvector for $A$ belonging to the eigenvalue $\lambda$ if

$$
A \mathbf{v}=\lambda \mathbf{v} \text { and } \mathbf{v} \neq 0 .
$$

The lengths of these eigenvectors are arbitrary, and we normalize them to unit length to obtain an orthonormal basis in three-dimensional space. Each eigenvector represents the unit normal to a plane, and the corresponding eigenvalue gives the sum of the squared normal deviations of the data from that plane. The minimum eigenvalue defines the plane of best leastsquares fit to the data, and the higher eigenvalues are associated with the remaining mutually orthogonal planes through the origin. With the origin fixed, the eigenvector basis represents a coordinate system that is rotated relative to the coordinate axes.

The eigenvectors written in columns form the matrix $P$. The elements of $P$ are the direction cosines between each eigenvector and the coordinate axes. The angle $\alpha_{i j}$ between the eigenvector $\mathbf{v}_{j}$ and the axis $\mathbf{i}_{i}$ is

$$
\alpha_{i j}=\cos ^{-1}\left(\mathbf{v}_{j} \cdot \mathbf{i}_{i}\right)=\cos ^{-1}\left(p_{i j}\right) .
$$

Because the columns of $P$ are orthonormal, $P$ is an orthogonal matrix, $P^{-1}=P^{T}$ is also orthogonal, and $\operatorname{det} P= \pm 1$. Matrices $A$ and $B$ are related through $P$ as

$$
A=P B P^{T}
$$

representing a singular value decomposition of $A$. The 
diagonal elements of $B$ are the singular values $\sigma_{i}$ as well as the eigenvalues of $A$. With singular values ordered by their magnitude in the same way as the eigenvalues, the condition number of matrix $A$ of full rank is

$$
\operatorname{cond}(\mathrm{A})=\frac{\sigma_{3}}{\sigma_{1}}=\frac{\lambda_{3}}{\lambda_{1}} \text {. }
$$

Condition numbers $\gg 1$ indicate that $A$ is nearly singular.

The sum of the squared normal distances between the data and the best plane, given by $\lambda_{1}$, provides a measure of the planar structure of an ice sample. Values of $\lambda_{1}$ or $\lambda_{1}^{\prime}$ approaching zero indicate an increasingly planar ice structure. If $\lambda_{1}=0$, then $\operatorname{cond}(A)=\infty$ and the data are perfectly planar. A visual representation of the error is obtained from the angle $\alpha_{m}$ between the $c$-axis of an individual crystal and its projection in the best plane (Fig. 2),

$$
\alpha_{m}=\left|\pi / 2-\cos ^{-1}\left(\mathbf{u}_{m} \cdot \mathbf{n}\right)\right| .
$$

The absolute value in Equation (32) is needed if the angle between $\mathbf{u}_{m}$ and $\mathbf{n}$ is greater than $\pi / 2$. The average angular deviation $\bar{\alpha}$ between the data and the best plane is a parameter we term the planar spread that can be readily determined and understood:

$$
\bar{\alpha}=\frac{1}{N} \sum_{m=1}^{N} \alpha_{m} .
$$

A small planar spread indicates a small mean angle between the $c$-axes of crystals in a sample and the plane.

A development that parallels the above to find the best plane by classical dependent variable least squares has been given by Ferrick and Claffey (1992). This standard method seeks a minimum error in the dependent variable and is more commonly available than the orthogonal method, but the results are sensitive to the choice of dependent variable. The normal distances between the data and the plane taking $z$ as the dependent variable can be determined as

$$
\left|\tilde{\mathbf{w}}_{m}\right|=\frac{\left(c_{1} x_{m}+c_{2} y_{m}-z_{m}\right)}{\left(c_{1}^{2}+c_{2}^{2}+1\right)^{\frac{1}{2}}} \text { for } m=1,2, \ldots, N .
$$

The sum of the squared normal distances given in Equation (34) is the same measure as the minimum eigenvalue. Similar expressions result when $x$ or $y$ is taken as the dependent variable.

\section{ALIGNMENT OF THE $c$-AXES}

An area of highly concentrated $c$-axis data on the Schmidt net indicates a linear preferred orientation of the ice crystals of a sample, and suggests the need to determine the predominant optic-axis orientation of the fabric. We will locate this linear orientation by following a development parallel to that used to determine the best plane. The unit vector c represents the unknown preferred $c$-axis orientation of the crystals in a sample, and is expressed as

$$
\mathbf{c}=c_{1}^{\prime} \mathbf{i}_{1}+c_{2}^{\prime} \mathbf{i}_{2}+c_{3}^{\prime} \mathbf{i}_{3}
$$

where the primes distinguish these coefficients from those of the unit vector $\mathbf{n}$ given in Equation (6). The plane through the origin that is normal to c represents the predominant basal plane orientation of the crystals in the sample and is described by

$$
F\left(x, y, z ; c_{1}^{\prime}, c_{2}^{\prime}, c_{3}^{\prime}\right)=c_{1}^{\prime} x+c_{2}^{\prime} y+c_{3}^{\prime} z=0 .
$$

As before, the unit vector $\mathbf{u}_{m}$ represents the $m$ th crystal $c$ axis orientation and intersects the unit hemisphere at $\left(x_{m}, y_{m}, z_{m}\right)$. In order to take advantage of the detail given in the previous development, the vector projections of $\mathbf{u}_{m}$ on to the plane and its normal vector are again $\tilde{\mathbf{v}}_{m}$ and $\tilde{\mathbf{w}}_{m}$, respectively. Then, with $\mathbf{c}$ replacing $\mathbf{n}$ and a different plane, Figure 2 represents our present condition.

When searching for the plane of best fit, we sought to minimize the squared normal distance. However, the closest representation of $\mathbf{u}_{m}$ by the unit vector $\mathbf{c}$ requires that we maximize the sum of the squares of the lengths of $\tilde{\mathbf{w}}_{m}$. The predominant basal plane orientation is then the plane of maximum squared normal error with the data. Each step in the previous development applies except that $c_{i}$ is replaced by $c_{i}^{\prime}$. Note that the matrix $A$ given in Equation (22) is unchanged because it depends only on the coefficients of the intersections of the individual unit vectors $\mathbf{u}_{m}$ with the unit hemisphere. Therefore, we are solving the same eigenvalue problem as before. The eigenvectors obtained in Equation (28) are orthogonal, and $\mathbf{c}$ is contained in the best plane. Kass (1989) has shown for the general case that spaces of closest fit are nested. Every $p$-dimensional sub-space of closest fit lies in one of dimension $p+1$. The third eigenvector $\mathbf{s}$ together with $\mathbf{c}$ form the plane of best fit to the $c$-axes, and $\mathbf{s}$ and $\mathbf{n}$ form the predominant basal plane (Fig. 3). The direction cosines of the angles between the preferred linear orientation and the coordinate axes are given in matrix $\mathrm{P}$, and the angles can be obtained from Equation (29).

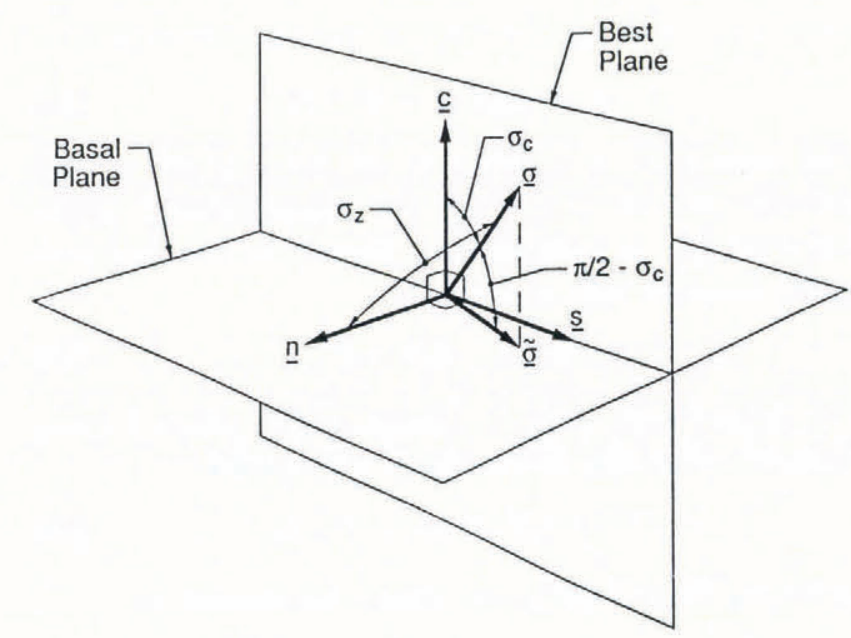

Fig. 3. Unit vector $\mathbf{c}$ representing the linear preferred caxis orientations of the ice fabric, and the long axis of the columnar crystals of an ice sample are represented by $\mathbf{n}$. The unit vectors $\mathbf{n}$ and $\mathbf{s}$ provide the predominant orientation of the basal plane of the sample. A unit vector $\sigma$ represents the direction of load application on the sample, $\tilde{\sigma}$ is the projection of this vector on to the basal plane, $\sigma_{c}$ is the angle between the applied load and $\mathbf{c}$, and $\sigma_{z}$ is the angle between the load and $\mathbf{n}$. 
The mean squared normal distance between the data and the vector $\mathbf{c}$ is $\lambda_{1}{ }^{\prime}+\lambda_{2}^{\prime}$ or $1-\lambda_{3}^{\prime}$. Again, using angles to visualize error, the angle $\delta_{m}$ between an individual unit vector and its projection on to $\mathbf{c}$ (Fig. 2 ), always $\leq 90^{\circ}$, is obtained as

$$
\delta_{m}=\cos ^{-1}\left(\mathbf{u}_{m} \cdot \mathbf{c}\right) .
$$

If $\delta_{m}$ obtained with Equation (37) is $>90^{\circ}, \delta_{m}$ is replaced by its supplement. The average angle $\bar{\delta}$ between the data and $\mathbf{c}$ is

$$
\bar{\delta}=\frac{1}{N} \sum_{m=1}^{N} \delta_{m}
$$

The parameter $\bar{\delta}$ is termed the linear spread, and a small $\bar{\delta}$ indicates that the angles between the preferred orientation and the data are also small.

As a group, the eigenvalues provide measures of the structure of the ice sample. An eigenvalue of zero occurs if the data are two-dimensional, and a pair of zero eigenvalues represent one-dimensional data. More generally, small $\lambda_{1}^{\prime} \approx \lambda_{2}^{\prime}$ together with large $\lambda_{3}^{\prime}$ occurs with aligned data, and small $\lambda_{1}^{\prime}$ with significantly larger $\lambda_{2}^{\prime}$ and $\lambda_{3}{ }^{\prime}$ indicates planar data. If, in addition to small $\lambda_{1}^{\prime}, \lambda_{2}^{\prime} \approx \lambda_{3}^{\prime}$, the crystal orientations distribute symmetrically about a great circle of the sphere. With $\lambda_{1}^{\prime}=\lambda_{2}^{\prime}=\lambda_{3}^{\prime}$ every set of orthonormal vectors will serve as the eigenvectors. The data are maximally dispersed with respect to lines and planes, and the material is isotropic.

The eigenvalue/eigenvector analysis has been applied to fabrics displaying other patterns, including multimaxima and small-circle girdles. Fitting these data with a best line or plane yields results that can only be qualitatively interpreted. However, when a fabric displays several point maxima, it may be possible to objectively subdivide the data. The best line for each sub-set would quantify the orientation and alignment of the corresponding point maximum. The intersection of cones with orientation diagrams was drawn by Kohnen and Gow (1979) to bound small-circle girdle fabrics. A small-circle girdle fabric could be assessed quantitatively by finding a circular cone with its axis defined by the best line and its apex at the origin. The surface of the cone would be located to minimize the sum of the squared distances with the data, and variability measures could again be defined in terms of distance and angle.

If the $c$-axes in a given sample are sufficiently aligned, the mechanical properties of the ice will be affected. If $\sigma$ is a unit vector in the direction of an applied force, the angle $\sigma_{c}$ between the load and the dominant $c$-axis direction is

$$
\sigma_{c}=\cos ^{-1}(\sigma \cdot \mathbf{c})
$$

and the complement of $\sigma_{c}$ is the angle between the load and the basal plane (Fig. 3). For a columnar ice sample, $\mathbf{n}$ gives the predominant direction of crystal elongation and growth. The angle $\sigma_{z}$ between the load and the vector $\mathbf{n}$ is obtained as

$$
\sigma_{z}=\cos ^{-1}(\sigma \cdot \mathbf{n})
$$

\section{SCHMIDT-NET REPRESENTATIONS}

The details necessary for automated plotting of the data, the plane of best fit and its pole, and vector of best fit on the Schmidt net in the $x y$-plane have been given by Ferrick and Claffey (1992). The intercept of $\mathbf{c}$ must fall on the great circle of best planar fit.

During field ice coring and thin-section preparation, a sample intended as horizontal may deviate by an angle of several degrees. Sea ice with horizontal $c$-axis alignment will then be represented by a best plane at this angle. The linear dimensions on the periphery of the Schmidt net are distorted, and it is difficult to judge normal distances between the data in this region and the trace of the best plane. Points that appear near the perimeter and directly across the net from each other represent crystals with close planar alignment. For these reasons, viewing the data on Schmidt nets drawn on alternative planes is frequently advantageous. Viewing the data on a Schmidt net drawn in the best-fit plane with $\mathbf{n}$ vertical eliminates sample-preparation error for horizontally aligned sea ice. The great circle of the best plane falls on the perimeter of the net and the pole of this plane appears at the origin. With c vertical, the plane of the Schmidt net is the predominant basal plane of the sample, and the data are transformed from the perimeter to the middle of the net. The great circle of the plane of best fit must then pass through the origin. Accurate visual assessments of the linear and planar preferred orientations of the sample are possible on this net because of minimal distortion of linear distance near the origin. With these planes for mapping, the importance of net distortion, sample preparation and measurement accuracy are minimized by providing optimal views of the crystal fabric.

Unit vectors in the Cartesian coordinate directions were used in Equation (7) to obtain a unit vector representing each crystal in a sample. These unit vectors are related to an orthogonal coordinate system of eigenvectors $\left(\mathbf{i}_{1}{ }^{\prime}, \mathbf{i}_{2}{ }^{\prime}, \mathbf{i}_{3}{ }^{\prime}\right)$ by the matrix $\mathrm{P}$ as

$$
\mathbf{i}_{j}^{\prime}=p_{i j} \mathbf{i}_{i}
$$

This relationship is depicted in Figure 4 with eigenvectors $\mathbf{n}, \mathbf{c}$ and $\mathbf{s}$ as the (primed) unit eigenvectors. The relationship between a general vector in the Cartesian system and its transform in the eigenvector system is (Hildebrand, 1965)

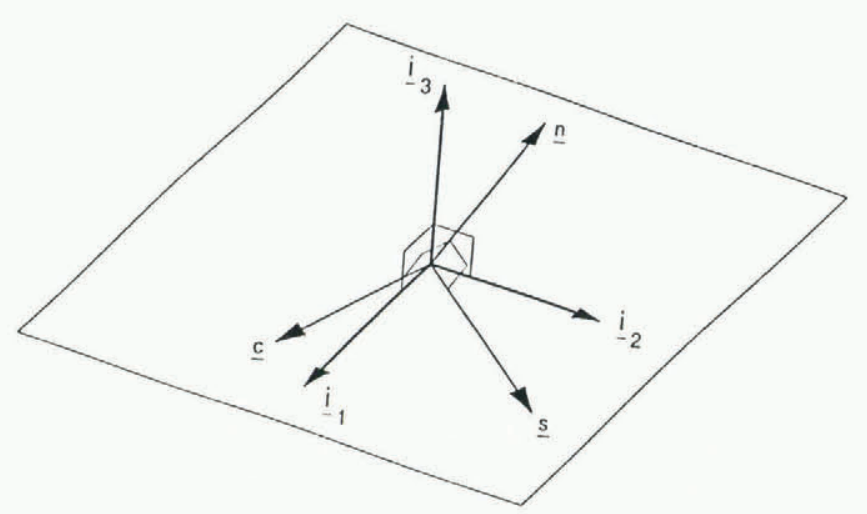

Fig. 4. Relationship between the Cartesian and eigenvector coordinate systems for visualizing alternate Schmidt-net representations of ice-fabric data. 


$$
\mathbf{u}_{m}^{\prime}=P^{-1} \mathbf{u}_{m}=P^{T} \mathbf{u}_{m}
$$

and $P$ is termed the transformation matrix. Because $P$ is orthogonal, transformations using $P$ are orthogonal. Orthogonal transformations maintain length and preserve angle, and can be interpreted as a combination of rotations and reflections. The order of the eigenvector placement in $P$ determines the transformation, and $\mathbf{n}$ or $\mathbf{c}$ is made vertical.

\section{APPLICATIONS}

We analyzed the ice fabric of many samples of first-year sea ice taken from the Beaufort Sea. Both orthogonal and dependent-variable least-squares analyses were used and compared. These cases are presented in Figure 5 in an arbitrary order from highest to lowest mean normal error of the best plane obtained from the orthogonal analysis. In all cases, this approach provided a lower bound to the normal error of the dependent-variable methods. The individual samples are designated by the dependentvariable analysis with mean normal error closest to that of the orthogonal analysis. The dashed lines connecting the mean normal errors for the dependent-variable solutions are erratic. Different dependent-variable choices produced dramatically different planes with widely varying normal errors. The coordinate axis most nearly normal to the plane of the data provided the lowest mean error. Diminishing mean normal error generally corresponds to diminishing planar spread. However, the minimizations of these two parameters are not equivalent, explaining the lack of perfect agreement between the trends plotted in Figure 5. The mean normal errors for most of these cases are less than 0.1 , corresponding to planar spreads of less than $12^{\circ}$.

The same cases were also analyzed for preferred alignment of the $c$-axes. Mean squared normal distance between the data and the best vector $\mathbf{c}$ is $1-\lambda_{3}^{\prime}$. The maximum normalized eigenvalue and the linear spread are displayed in Figure 6. Values of $\lambda_{3}^{\prime}$ that exceed 0.9 correspond to linear spreads of less than $15^{\circ}$. Linear spread is larger than planar spread because it represents angles with a particular line in the plane, while planar spread represents angles with the plane itself. Comparing Figures 5 and 6 , we observe that increasingly planar seaice fabrics do not necessarily correspond to the degree of preferred alignment.

Schmidt-net plots of the data representing cases $z_{1}, z_{10}^{T}$ and $z_{11}$ are presented in Figure 7 and parameters developed from these data are given in Table 1 . Included on each net are the intersections with the hemisphere of the plane of best fit, the pole $P$ of this plane, and the vector $\mathbf{c}$ of best linear fit. The best line is always contained in the best plane. In case $z_{1}$, the normalized eigenvalues are approximately equal, the planar and linear spreads are large, and the matrix $\mathrm{A}$ is non-singular. These conditions indicate that the data are uniformly distributed over the surface of the sphere. Cases $z_{10}^{T}$ and $z_{11}$ are very different from $z_{1}$, but similar to each other. For this

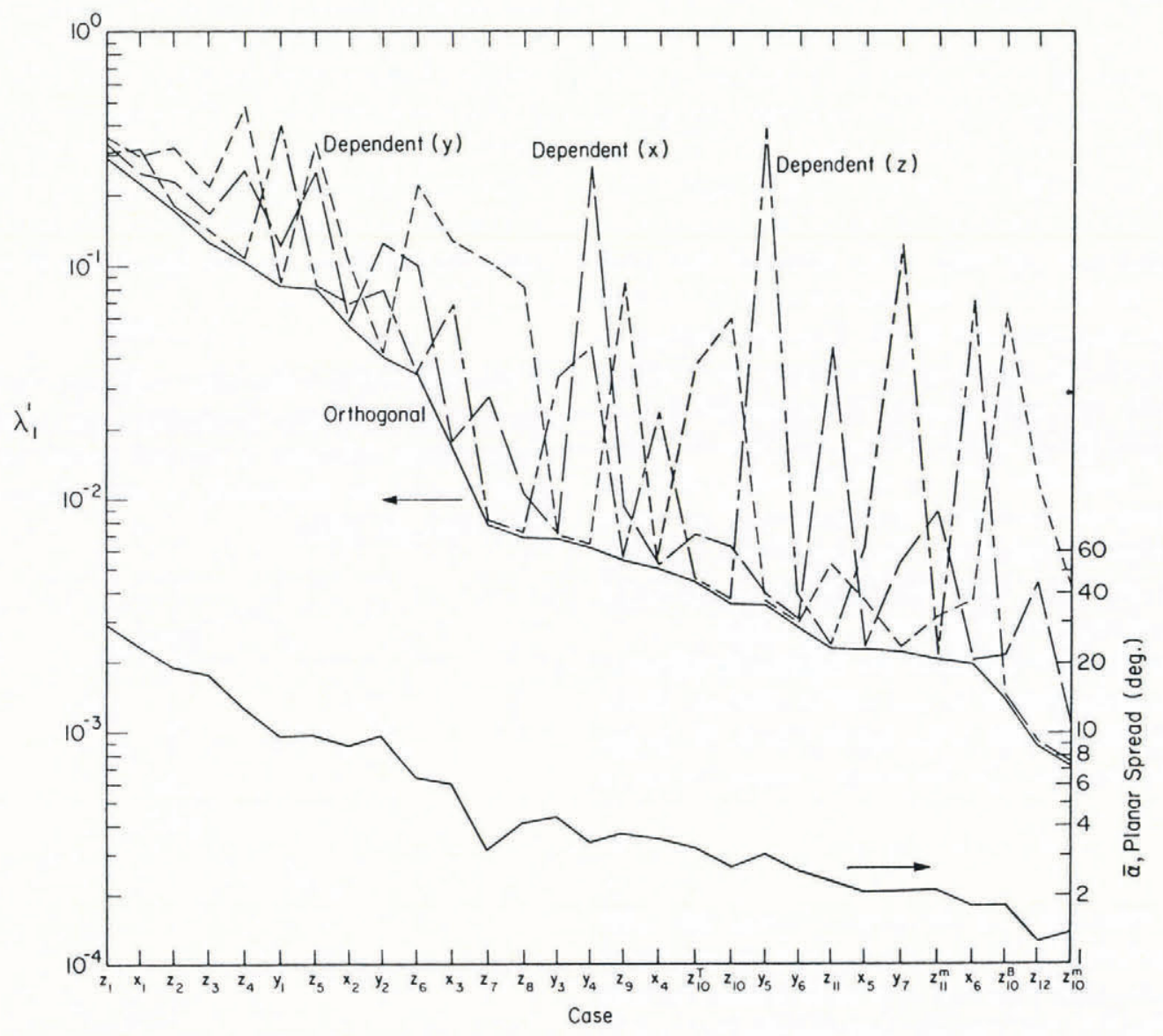

Fig. 5. Mean squared normal error for several sea-ice samples from orthogonal and $x$-, $y$ - and $z$-dependent variable leastsquares analyses. Planar spread from the best plane is given for these same cases. The cases are arranged arbitrarily according to $\lambda_{1}^{\prime}$ of the orthogonal analysis and named in sequence according to the best dependent-variable solution. 


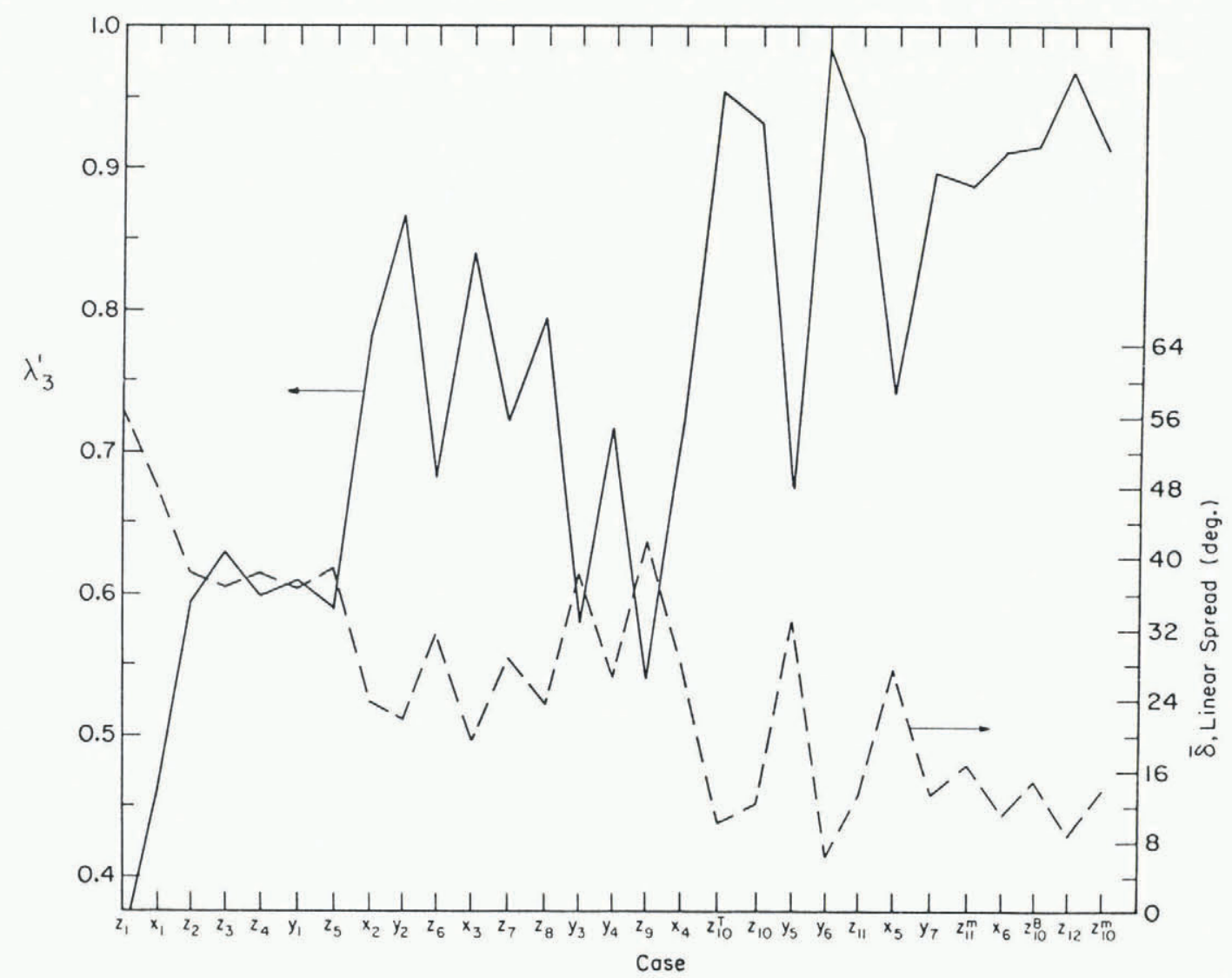

Fig. 6. Maximum normalized eigenvalue and linear spread in degrees for several sea-ice samples. The order and labeling of the cases is the same as in Figure 5.

group of samples $\lambda_{1}$ is smallest for $z_{11}$, indicating that these data are the most closely represented by a plane. Sample $z_{10}{ }^{T}$ has the smallest $\lambda_{2}^{\prime}$ value together with a small $\lambda_{1}^{\prime}$ and is the most linear case in this group. The distribution of data indicated by the eigenvalues is confirmed by the angles that quantify the planar and linear spreads $\bar{\alpha}$ and $\bar{\delta}$, respectively, for each sample. The matrix A is nearly singular in the latter two cases as $\lambda_{1} \approx 0$ and the condition number is large.

A group of samples taken in close proximity and from the same vertical position in the ice sheet should have similar structure. Together, the individual and collective

Table 1. Normalized eigenvalues and condition number of matrix $\mathrm{A}$, and planar and linear spread of the data for selected cases. The type of the distribution is interpreted from these parameters

$\begin{array}{llllllllll}\text { Case } & \mathcal{N} & \lambda_{1}^{\prime} & \lambda_{2}^{\prime} & \lambda_{3}^{\prime} & \bar{\alpha} & \bar{\delta} & \text { Cond }(A) & \begin{array}{c}\text { Type of } \\ \text { distribution }\end{array}\end{array}$

$\operatorname{deg} \quad \operatorname{deg}$

$\begin{array}{lllllllll}z_{1} & 90 & 0.294 & 0.351 & 0.355 & 29.3 & 56.4 & 1.21 & \text { Uniform } \\ z_{11} & 77 & 0.00235 & 0.0755 & 0.922 & 2.3 & 13.5 & 392 & \text { Planar/linear } \\ z_{10}{ }^{T} & 44 & 0.00452 & 0.0417 & 0.954 & 3.2 & 10.4 & 211 & \text { Linear/planar } \\ z_{10}{ }^{M} & 22 & 0.00073 & 0.0863 & 0.913 & 1.4 & 14.2 & 1250 & \text { Planar/linear } \\ z_{10}{ }^{B} & 23 & 0.00140 & 0.0813 & 0.917 & 1.8 & 14.9 & 653 & \text { Planar/linear } \\ z_{10} & 89 & 0.00359 & 0.0638 & 0.933 & 2.7 & 12.5 & 260 & \text { Planar/linear }\end{array}$




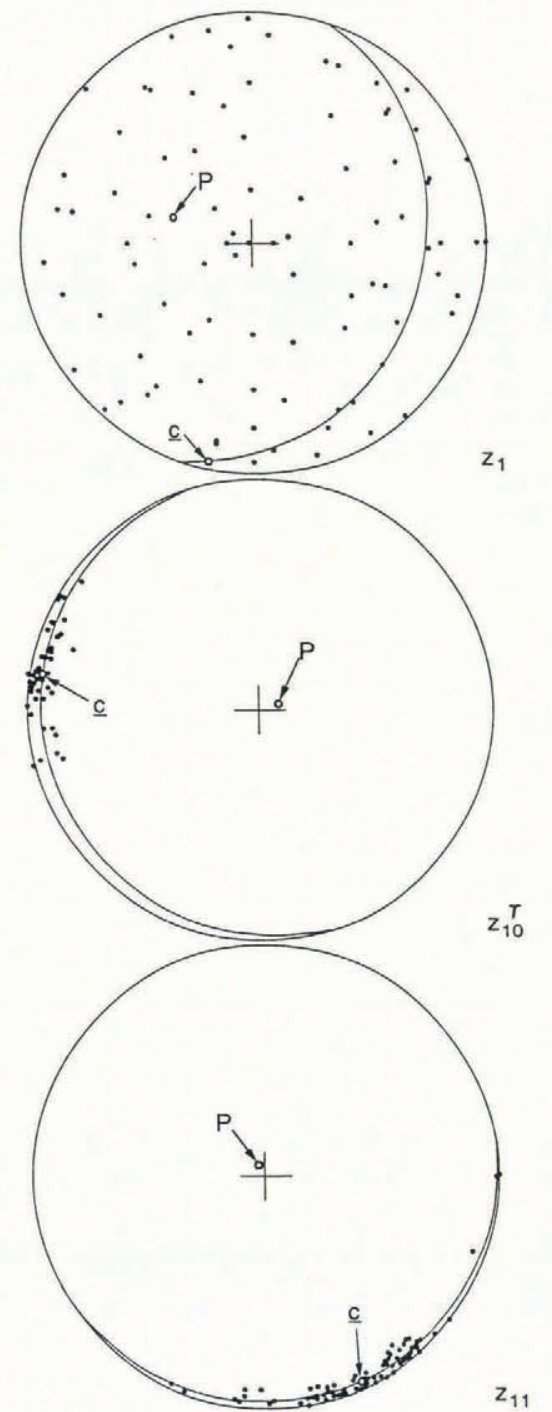

Fig. 7. Schmidt-net plots of cases $z_{1}, z_{10}{ }^{T}$ and $z_{11}$ including the intersections with the hemisphere of the best plane, the pole $P$ of this plane, and the best vector $\mathbf{c}$.

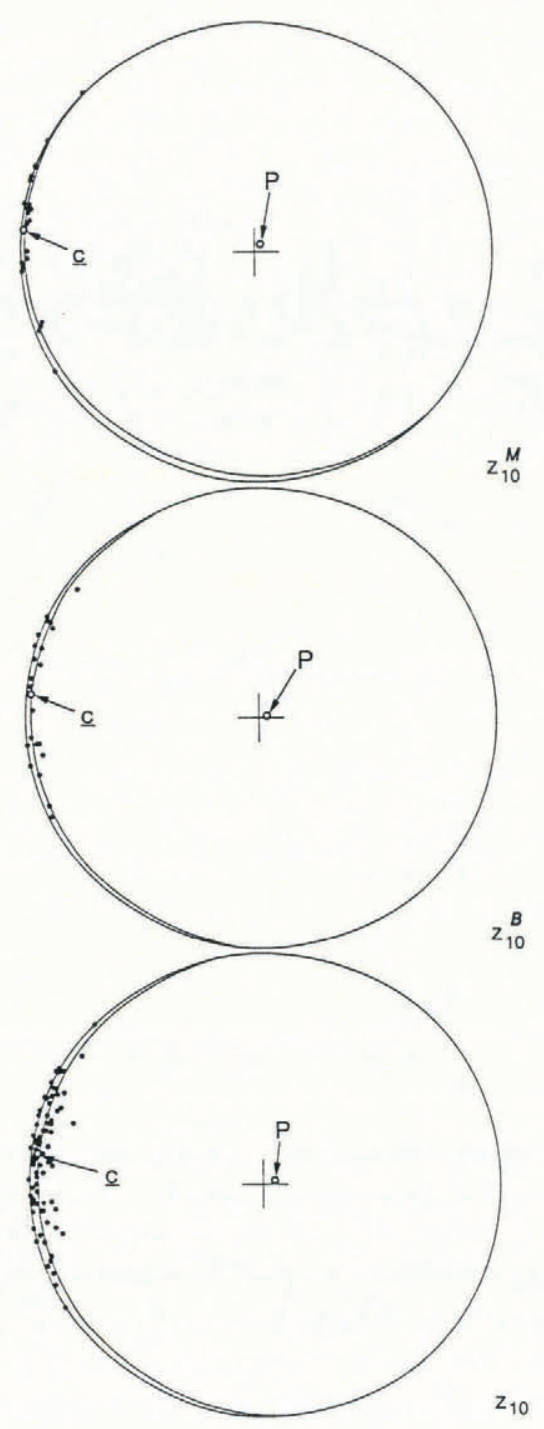

Fig. 8. Schmidt-net plots of cases $z_{10}{ }^{M}, z_{10}{ }^{B}$ and composite case $z_{10}$ including the intersections with the hemisphere of the best plane, the pole $P$ of this plane, and the best vector $\mathbf{c}$. analyses of these samples provide quantitative measures of comparison that indicate the sample size needed to represent the ice fabric at that location. Three samples designated $z_{10}{ }^{T}, z_{10}{ }^{M}, z_{10}{ }^{B}$ were taken from an ice core at $1.3 \mathrm{~m}$ from the surface. The Schmidt-net plots of both the data and the computed fits are presented in Figure 7 for $z_{10}^{T}$ and in Figure 8 for the other samples and the composite data. Each of these cases has nearly the same planar structure, pole and linear structure. This similarity is quantified by the eigenvalues, planar spreads and linear spreads given in Table 1 .

All the Schmidt nets presented above depict the lower hemisphere on the $x y$-plane. Alternative Schmidt nets are obtained for the hemispheres below the best fit and predominant basal planes using Equation (42) and a $\mathrm{P}$ matrix with different eigenvector placement in each case. As a result of these transformations, the position of the data on the net shifts, correcting for sample-preparation error and allowing a visual assessment of the distances from the points to the best line and plane. Figure 9 gives the $x y$ and the alternative nets for sample $z_{10}{ }^{T}$. The data are near the perimeter of the net in the standard $x y$-plot.
The net in the best plane displays a balanced distribution of data on opposite sides of the net. The net in the basal plane depicts the points as a single group near the center of the net where linear distance is accurately represented and the linear and planar fits can be readily evaluated and compared to other samples.

\section{CONCLUSIONS}

A quantitative characterization of ice fabrics is critical for understanding the mechanical properties of sea ice, but was not previously available. An orthogonal least-squares analysis of uniaxial crystal-orientation data was developed from geometric arguments with unit vectors representing individual crystal orientations. Minimization of the perpendicular distances with a best line or plane provided an eigenvalue problem that was identical to that obtained by other investigators using different methods. Normalized eigenvalues give the mean squared normal distance of the data from the line or plane, and corresponding eigenvectors provide the dominant $c$-axis, 


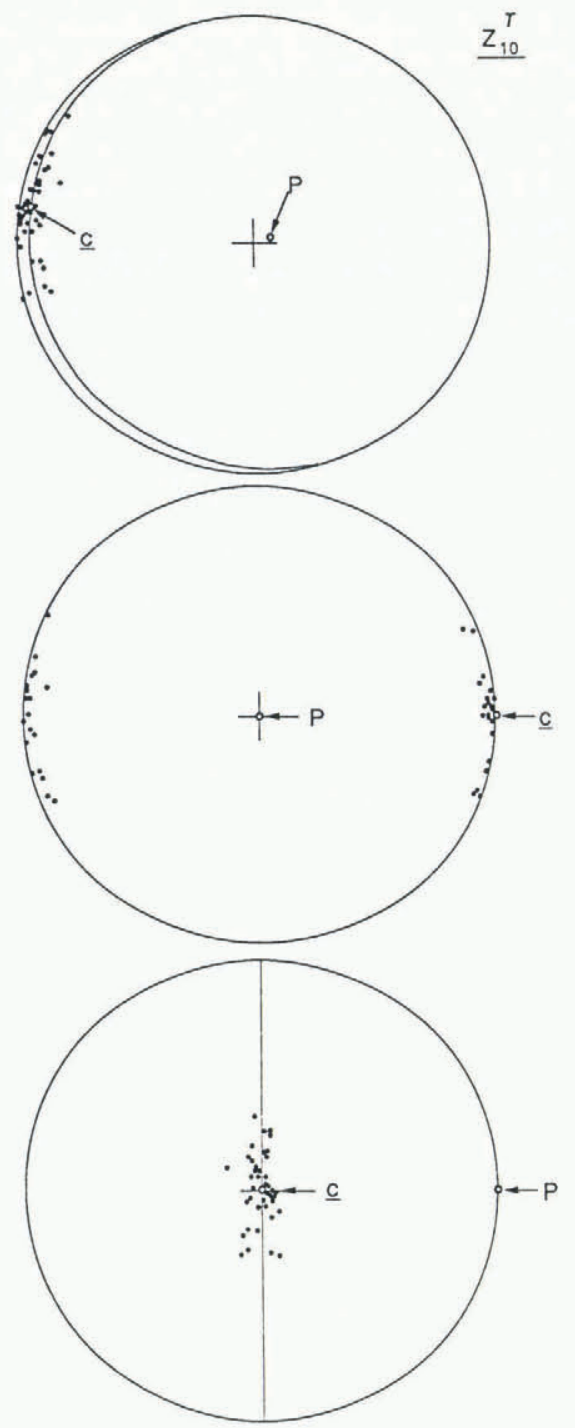

Fig. 9. Schmidt-net plots of case $z_{10}^{T}$ in the standard $x y$ plane with the $z$-axis vertical, in the best plane with $\mathbf{n}$ vertical, and in the predominant basal plane with $\mathbf{c}$ vertical.

planar and basal plane orientations, and the direction of columnar-crystal elongation. The preferred $c$-axis orientation is always contained in the plane of best fit. The method is the basis of a relatively simple algorithm for computer analysis of large volumes of orientation data.

The formulation of a least-squares method greatly influences the results. This observation was demonstrated for many samples of first-year sea ice by comparing the mean squared normal distance of the data with planes obtained using the classical dependent-variable leastsquares approaches and the orthogonal method. The dependent-variable solutions produced dramatically different planes of best fit with erratic and widely varying normal errors. The error approached the minimum given by the orthogonal method when the dependent-variable direction was almost normal to the best plane. Orthogonal least-squares and other analogous methods producing the eigenvalue problem all rely on the implicit assumption of equal measurement error in all coordinate directions at all points on the unit sphere. We find that this assumption is a first approximation for optical data obtained with the universal stage.

Normalized eigenvalues give the mean squared normal distance between the data on the unit sphere and the plane through the origin normal to the corresponding eigenvector. This measure of the planar fit to data is equivalent to the variance in classical dependent-variable least-squares methods. The majority of the sea-ice samples studied had planar fabrics and several had aligned fabrics, characterized by mean squared normal distances of less than 0.1 with the data. These relative distances are well represented by the angular measures of linear and planar spread; however, the distance and angular error measures are not equivalent. For these samples, increasingly planar orientations of the $c$-axes do not correspond to increasingly linear fabrics.

The normalized eigenvalues allow quantitative comparisons betwen samples, and of composite data representing a collection of several samples. Proximate samples from the same vertical position in the ice sheet had nearly identical structures. This similarity, quantified by the computed eigenvalues and eigenvectors, was displayed on Schmidt nets for the individual samples and the composite. The capability to view the data and the analytical results on Schmidt nets in the planes defined by the eigenvectors was developed, providing information to improve the interpretation of the data and the fits by minimizing the importance of sample preparation, net distortion and measurement accuracy limitations.

\section{ACKNOWLEDGEMENTS}

We should like to thank J. Richter-Menge for bringing the need for sea-ice-fabric analysis to our attention and for many helpful discussions on this topic. We thank D. Harp for patience and skill in preparing many drafts, E. Wright for careful editing, and M. Hopkins and A.J. Gow for insightful reviews.

\section{REFERENCES}

Beyer, W. H. 1987. Handbook of mathematical sciences. Sixth edition. Boca Raton, FL, CRC Press.

Diggle, P.J. and N. I. Fisher. 1985. Sphere: a contouring program for spherical data. Comput. Geosci., 11(6), 725766.

Fairbairn, H. W. 1949. Structural petrology of deformed rocks. Cambridge, MA, Addison-Wesley Publishing Co.

Ferrick, M. G. and K.J. Claffey. 1992. Vector analysis of ice fabric data. CRREL Rep. 92-1.

Herron, S. L. and C. C. Langway, Jr. 1982. A comparison of ice fabrics and textures at Camp Century, Greenland and Byrd Station, Antarctica. Ann. Glaciol., 3, 118-124.

Hildebrand, F.B. 1965. Methods of applied mathematics. Second edition. Englewood Cliffs, NJ, Prentice-Hall.

Kass, S. 1989. Spaces of closest fit. Linear Algebra and Its Applications, 117, 93-97.

Knopf, E. B. and E. Ingerson. 1938. Structural petrology. Geol. Soc. Am. Mem. 6.

Kohnen, H. and A.J. Gow. 1979. Ultrasonic velocity investigations of crystal anisotropy in deep ice cores from Antarctica. CRREL Rep. 79-10. 
Langway, C. C., Jr. 1958. Ice fabrics and the universal stage. SIPRE Tech. Rep. 62.

Mardia, K. V. 1972. Statistics of directional data. New York, Academic Press.

Pearson, K. 1901. On lines and planes of closest fit to systems of points in space. Philos. Mag., 6th Ser., 2(11), 559-572.

Reed, B. C. 1989. Linear least-squares fits with errors in both coordinates. Am. 7. Phys., 57(7), 642-646.

Richter-Menge, J.A., G.F. N. Cox and N.M. Perron. 1987. Mechanical properties of multi-year sea ice, Phase I. Ice structure analysis. CRREL Rep. 87-3.

Turner, F.J. and L. E. Weiss. 1963. Structural analysis of metamorphic tectonites. New York, McGraw-Hill Book Co.
Wang, Y.S. 1979. Crystallographic studies and strength tests of field ice in the Alaskan Beaufort Sea. In POAC 79; the Fifth International Conference on Port and Ocean Engineering under Arctic Conditions. ... Vol. 1., 651-665.

Watson, G. S. 1966. The statistics of orientation data. $\mathcal{J}$. Geol., 74(5), 786-797.

Weeks, W.F. and S.F. Ackley. 1982. The growth, structure, and properties of sea ice. CRREL Monogr. 82-1.

Weeks, W.F. and A.J. Gow. 1978. Preferred crystal orientations in the fast ice along the margins of the Arctic Ocean. 7. Geophys. Res., 83(C10), 5105-5121.

The accuracy of references in the text and in this list is the responsibility of the authors, to whom queries should be addressed. 